\title{
ASOCIACIÓN ENTRE LA CERVICOMETRÍA Y EL PARTO PREMATURO EN PACIENTES CON SOSPECHA DE TRABAJO DE PARTO PRETÉRMINO INICIAL
}

\author{
Associating measuring cervical length with preterm birth amongst hos- \\ pitalised women diagnosed with threatened preterm labour \\ Antonio González, M.D. *, Jorge Hernando Donado, M.D.**, \\ David Felipe Agudelo, M.D.***, Hernán Darío Mejía, M.D.***, \\ Claudia Bibiana Peñaranda, M.D.***
}

Recibido: enero 18/2005 - Revisado: marzo 3/2005 - Aceptado: mayo 12/2005

\section{RESUMEN}

Objetivo: determinar en gestantes que fueron hospitalizadas con diagnóstico clínico de trabajo de parto pretérmino (TPP) inicial, embarazo único entre las 20 y 36 semanas de gestación y membranas íntegras, la asociación de la cervicometría y otros factores de riesgo, con el parto prematuro por TPP idiopático en nuestra comunidad.

Metodología: estudio observacional analítico de tipo cohorte prospectiva realizado en los servicios de obstetricia de tres instituciones en el área metropolitana de Medellín, durante los meses de enero a junio de 2004. Las pacientes fueron divididas en dos grupos: el primero con cervicometría positiva (longitud cervical $<30 \mathrm{~mm}$ ) y un segundo grupo con cervicometría negativa (longitud cervical $\geq 30$ $\mathrm{mm}$ ) y se determinó la frecuencia de parto prematuro en cada uno de ellos.

Resultados: se estudiaron 156 pacientes de las cuales 22 fueron excluidas por no tener información acerca del resultado de la gestación. 53 tenían

* Ginecoobstetra. Asesor clínico. Profesor asociado, Universidad Pontificia Bolivariana. Medellín - Colombia. Correo electrónico: antoniogo@ cis.net.co

* Médico Internista y Magíster en Epidemiología. Asesor Epidemiológico. Profesor asociado, Universidad Pontificia Bolivariana. Medellín.

*** Residentes de III año de Ginecoobstetricia. Facultad de Medicina, Universidad Pontificia Bolivariana 2004. Medellín. cervicometría positiva y 81 cervicometría negativa. En el análisis bivariado del parto prematuro con las variables independientes estudiadas, sólo la longitud del cérvix menor $30 \mathrm{~mm}$ y la infección vaginal tuvieron asociación estadísticamente significativa. $\mathrm{La}$ única variable que en presencia de todas las demás presentó un valor estadísticamente significativo en el análisis multivariado fue la longitud cervical menor de $30 \mathrm{~mm}$ con OR 11,1 (IC95\% 4,55 - 27,05).

Conclusiones: en la población en estudio, la detección en la cervicometría de una longitud cervical menor de $30 \mathrm{~mm}$ se constituye en un factor asociado a la posibilidad de tener un parto prematuro como desenlace final.

Palabras clave: trabajo de parto pretérmino, cuello uterino, ultrasonografía, parto prematuro.

\section{SUMMARY}

Objective: assessing the accuracy of measuring cervical length when predicting preterm birth amongst a population of hospitalised women diagnosed with threatened preterm labour (initial preterm labour), single pregnancy and intact foetal membranes.

Method: a prospective cohort study was carried out of the obstetrics services of three institutions from the metropolitan area of Medelín between January and June 2004. The sample size was estimated 
with a 0.05 alpha, $80 \%$ power, $2.5 \%$ preterm birth incidence in the unexposed group and 6.0 relative risk. There were 76 patients in two groups (152 patients in all). The first group of 76 patients had a positive cervical length (less than $30 \mathrm{~mm}$ cervical length). The second group had a negative cervical length (cervical length of more than $30 \mathrm{~mm}$ ).

Results: 156 hospital charts were reviewed; 22 were excluded because they did not contain information regarding final gestation outcome. 53 out of the remaining 134 charts presented a positive cervical length (less than $30 \mathrm{~mm}$ ) and 81 had negative cervical length (more than $30 \mathrm{~mm}$ ). Bivariable analysis of the main final outcome (preterm birth) revealed that the only statistically significant outcomes were having a cervical length of less than $30 \mathrm{~mm}$ and vaginal infection $(p<0.05)$. The only statistically significant outcome (when using multivariable analysis) was having a cervical length of less than $30 \mathrm{~mm}$ (11.1 OR, 4.55-27.05 95\% CI).

Conclusions: cervical length of less than $30 \mathrm{~mm}$ was a risk predictor in the study population for preterm labour in patients diagnosed with initial preterm labour.

Key words: premature labour, cervix, uterineultrasonography.

\section{INTRODUCCIÓN}

El parto prematuro es la principal causa de mortalidad y morbilidad perinatal. El parto prematuro espontáneo incluye los nacimientos entre las 20 y 37 semanas producto de un trabajo de parto pretérmino idiopático, ruptura espontánea de membranas ovulares pretérmino e incompetencia cervical. El parto prematuro por indicación se produce por la necesidad médica de anticipar el nacimiento ante un riesgo materno, fetal o ambos. ${ }^{1}$ El 75\% de la mortalidad perinatal, excluyendo la atribuible a malformaciones congénitas, es producto de los nacimientos prematuros. Aunque en los países más desarrollados se ha logrado una reducción importante en la mortalidad, aún hoy persisten altas tasas de morbilidad y secuelas neurológicas. En Estados
Unidos la incidencia de parto prematuro en 1981 fue de $9,4 \%$ y las cifras muestran un aumento progresivo a $10,6 \%$ en $1990,11,6 \%$ en 2000 y $11,9 \%$ en 2001. ${ }^{2}$ En otros países, por el contrario, la incidencia del parto prematuro se ha reducido, como en Alemania, país donde cayó del 7\% registrado en 1981 , a $4 \%$ en $1989 .^{3}$

El trabajo de parto pretérmino (TPP) idiopático se define como la presencia de contracciones uterinas regulares acompañadas de cambios cervicales que acontecen entre la semana 20 y la 37 de gestación; se considera como inicial cuando se detecta en fase latente (en nuestro medio se denomina "amenaza de parto prematuro"). La presencia de contracciones uterinas sin cambios cervicales ("irritabilidad uterina") es difícil de definir como entidad y la detección de cambios cervicales sin contracciones sugiere incompetencia cervical.

El TPP idiopático es causa del 40 - 50\% de todos los partos prematuros; la ruptura prematura de las membranas ovulares y la incompetencia cervical causa del 25 al $40 \%$ y el parto prematuro indicado explica el 20 - 25\% restante. ${ }^{4}$ Aunque hay muchas condiciones maternas asociadas con el TPP idiopático, la etiología en la mayoría de los casos no es clara. Actualmente se considera como un síndrome, es decir, una condición desencadenada por múltiples causas, generalmente coexistentes, que se expresan finalmente con contracciones uterinas y cambios cervicales. El antecedente de parto prematuro es un factor de riesgo importante para que se presente un nuevo parto prematuro en gestaciones subsecuentes (OR 6). Se ha reportado que este riesgo se correlaciona con el número de partos prematuros anteriores, $\mathrm{y}$ tiende a incrementarse fuertemente a menor edad del parto prematuro anterior $(<$ de 32 semanas). Otros factores asociados al TPP son las edades extremas, la raza negra, el tabaquismo, las infecciones vaginales bacterianas, la infección del tracto urinario y otros..$^{5-7}$

Se han realizado múltiples esfuerzos para prevenir el TPP idiopático; las diversas medidas implementadas no han probado ser efectivas. Igualmente 
frustrantes han sido las diferentes modalidades terapéuticas que se han instaurado en pacientes sintomáticas para intentar contrarrestarlo y prolongar su gestación. ${ }^{8}$

Existe la necesidad de encontrar medidas eficaces de detección y evaluación del TPP idiopático inicial, a fin de definir qué pacientes requieren intervención y disminuir los altos costos derivados de la atención de la madre y del recién nacido prematuro, así como la morbilidad y mortalidad neonatal.

Durante mucho tiempo la prueba de referencia para el diagnóstico de TPP inicial fue la documentación de los cambios cervicales por medio del examen digital en presencia de contracciones uterinas regulares. En la última década se han descrito nuevas pruebas bioquímicas y biofísicas, como la cervicometría por ultrasonido vaginal, que han demostrado superioridad frente al examen digital. ${ }^{9}$ Los hallazgos observados mediante el ultrasonido transvaginal del cuello uterino permiten entender muchos de los cambios que se producen durante la gestación normal, y anticipar qué mujeres tendrán un mayor riesgo de TPP y parto prematuro. ${ }^{10}$

Por lo anterior, se quiso evaluar la medición de la longitud cervical y algunos factores de riesgo que pueden servir como elementos coadyuvantes en el diagnóstico acertado del TPP idiopático inicial.

\section{MATERIALES Y MÉTODOS}

Se realizó un estudio observacional analítico de tipo cohorte prospectiva en los servicios de obstetricia de la Clínica Universitaria Bolivariana (CUB), Empresa Social del Estado Rafael Uribe Uribe (Clínica León XIII) del Municipio de Medellín y en el Hospital Marco Fidel Suárez (HMFS) del Municipio de Bello entre enero y junio de 2004.

\section{Población y selección de la muestra}

Pacientes que consultaron al servicio de urgencias de una de las tres instituciones antes mencionadas con síntomas sugestivos de TPP idiopático y que fueron hospitalizadas para el manejo de su entidad. Las pacientes seleccionadas para el estudio inclu- yeron gestantes con un embarazo único, con edad gestacional confirmada mayor de 20 y menor de 37 semanas. Se excluyeron las gestantes sin cervicometría en las primeras 24 horas, embarazos múltiples, ruptura prematura de membranas antes o durante la hospitalización y trabajo de parto avanzado documentado por el tacto vaginal con dilatación mayor de $3 \mathrm{~cm}$ y/o longitud del cérvix menor de $1 \mathrm{~cm}$.

El tamaño de la muestra se estimó con un alfa 0,05 , poder del $80 \%$, incidencia de parto prematuro en el grupo no expuesto del 2,5\% y un riesgo relativo de 6,0, necesitando un total de 152 que fueron divididas en dos grupos: el primero con cervicometría positiva (longitud cervical $<30 \mathrm{~mm}$ ) y el segundo con cervicometría negativa (longitud cervical $\geq 30 \mathrm{~mm}$ ). Se realizó un muestreo secuencial por conveniencia.

Al momento del ingreso se confirmó la presencia de actividad uterina regular y mediante tacto vaginal se determinó el estado del cuello uterino en cuanto a dilatación y longitud. El médico a cargo consignó en la historia clínica los hábitos y antecedentes de las pacientes con énfasis en las variables elegidas para el estudio. En la sala de hospitalización se tomaron muestras de orina para citoquímico y uroanálisis, flujo vaginal con espéculo y en las 24 horas siguientes al ingreso, se hizo una valoración cervical mediante ultrasonido transvaginal en un equipo Siemens Sonoline SI - 250 con una técnica previamente estandarizada. Todas las pacientes recibieron dosis variables de un tocolítico endovenoso y betametasona intramuscular a dosis convencionales, reposo en cama e hidratación oral; el parto ocurrió durante o después de la hospitalización.

\section{Variables (definiciones operativas) Trabajo de parto pretérmino: (TPP)o amenaza} de parto prematuro. Se definió como la presencia de contracciones uterinas regulares y cuello uterino con dilatación menor de $3 \mathrm{~cm}$ y longitud mayor de $1 \mathrm{~cm}$ que acontecen después de las 20 semanas y antes de las 37 semanas de gestación y que obligó a la hospitalización de la gestante. 
Parto prematuro previo: antecedente de uno o más partos prematuros espontáneos.

Tabaquismo: consumo de 1 ó más cigarrillos al día.

Infección urinaria: documentada por citoquímico de orina anormal y/o urocultivo patológico.

Infección vaginal: documentada con examen directo y gram de flujo vaginal definido como normal $o$ anormal (o positivo para vaginitis $\mathrm{y} / \mathrm{o}$ vaginosis $\mathrm{y} / \mathrm{o}$ infección mixta).

Cervicometría: medida por ecografía transvaginal de orificio a orificio en un corte longitudinal del mismo que incluya la totalidad del conducto cervical; se consideró positiva si la longitud cervical fue menor a $30 \mathrm{~mm}$ con o sin un orificio interno mayor de 10 $\mathrm{mm}$, con o sin embudización de las membranas, y se consideró negativa si la longitud cervical fue $\geq$ $30 \mathrm{~mm}$ con o sin un orificio cervical interno mayor de $10 \mathrm{~mm}$, con o sin embudización.

Dilatación del orificio cervical interno: medida de la amplitud de la embudización de borde a borde en $\mathrm{mm}$ a nivel del orificio cervical interno mediante ecografía transvaginal; se consideró significativa al medir más de $10 \mathrm{~mm}$.

Parto prematuro: cualquier parto después de la semana 20 pero antes de las 37 semanas de gestación durante o después de la hospitalización.

\section{Plan de análisis}

Las características basales de los pacientes se presentan como números absolutos y proporciones para datos nominales y como media con desviación estándar para datos cuantitativos distribuidos normalmente o mediana y rango intercuartílico para datos cuantitativos no distribuidos normalmente.

Para las variables cuantitativas continuas se realizó la prueba de Kolmogorov - Smirnov para definir si su distribución es normal o no.

Se comparó la variable parto prematuro con cada una de las variables independientes mediante el uso de prueba $t$ - Student para datos paramétricos, prueba U de Mann Whitney para datos no paramétricos y el Ji cuadrado de Pearson o la prueba exacta de Fisher para variables cualitativas. Se estimó el riesgo relativo (RR).

Para establecer una asociación se empleó el análisis de regresión logística binaria, ajustando el efecto de las variables independientes sobre el desenlace. Se ingresaron en el modelo aquellas variables que tenían una asociación en el análisis bivariado con valor de $\mathrm{p}<0,25$ o bien que tenían plausibilidad biológica. Se evaluaron las distintas interacciones plausibles. Se realizó estudio de colinealidad entre las variables. La medida de asociación se calculó mediante el Odds Ratio (OR) y el intervalo de confianza del 95\%. Todas las pruebas de significancia estadística fueron de dos colas y con valor de $\mathrm{p}<0,05$. Para el manejo informático de los datos se utilizó el programa de computador SPSS 10 (SPSS Inc 10,0 for Windows Chicago, Illinois).

\section{Aspectos éticos}

Para la realización del estudio se tuvieron en cuenta todas las especificaciones en cuanto a la investigación en seres humanos establecidas por la Resolución 08430 del Ministerio de Salud Nacional de Colombia, además de los cánones establecidos por la Declaración de Helsinki enmendada en la 52 ${ }^{\mathrm{a}}$ Asamblea General Edimburgo, Escocia, octubre de 2000.

Se contó con la aprobación del Comité de Ética de la Universidad Pontificia Bolivariana y de las instituciones participantes. Se obtuvo el consentimiento informado de las pacientes incluidas en el estudio. Los autores no reportan ningún conflicto de interés.

\section{RESUITADOS}

En un corte se analizaron 134 pacientes de las cuales la mayoría, 62\% (83) pertenecían a la Clínica Universitaria Bolivariana (CUB). La distribución por edades tuvo una mediana de 25 años, con un rango intercuartílico entre los 20 y 30 años. La mediana de la edad gestacional al ingreso fue de 32 semanas; se encontró que la menor edad gestacional fue de $23+2 / 7$ semanas y la máxima de $36+5 / 7$ semanas. Discriminando por edad gestacional, se incluyeron 62 pacientes con menos de 32 semanas, 39 pacientes entre 32 y 34 sema- 
nas, 26 gestantes entre 34 y 36 semanas y 7 con embarazo mayor de 36 semanas.

En cuanto al número de gestaciones, la mediana fue de 2 con un rango intercuartílico entre 1 y 3 , siendo la menor gravidez de 1 y la máxima de 8 . Con relación a tener antecedente de parto prematuro en gestaciones anteriores, de las 134 pacientes, sólo 5 pacientes presentaron este antecedente $(3,7 \%)$.

En cuanto al antecedente de tabaquismo, de las 134 pacientes, solamente 3 eran fumadoras (2,2\%). En cuanto a la infección urinaria durante este embarazo, sólo 25 pacientes $(18,2 \%)$ refirieron el antecedente. Respecto al antecedente de infección vaginal durante el embarazo se presentó vaginosis o vaginitis en 23 pacientes (17,2\%).

La longitud del cuello por cervicometría tuvo una mediana de $36 \mathrm{~mm}$ con un rango intercuartílico entre 27,45 mm y 41,30 mm, siendo lo mínimo de dicha longitud $10 \mathrm{~mm}$ y lo máximo $56 \mathrm{~mm}$. A su vez la dilatación del orificio cervical interno $(\mathrm{OCI})$ por cervicometría tuvo una mediana de $0,0 \mathrm{~mm}$ con un rango intercuartílico entre 0,0 y 5,6 mm.

\section{Análisis bivariado de los factores de riesgo}

Del total de pacientes hospitalizadas con sospecha de TPP inicial (134), 45,5 \% (61) tuvieron un parto prematuro; la asociación de los factores de riesgo con el parto prematuro puede observarse en la tabla $\mathbf{1}$.

En el análisis bivariado de la variable parto prematuro con las variables independientes estudiadas sólo la infección vaginal y la longitud del cérvix menor $30 \mathrm{~mm}$ tuvieron asociación estadísticamente significativa (valor de $\mathrm{p}<0,05)$ (tabla 1 ).

La tabla 2 muestra la relación entre la longitud cervical y el riesgo de prematurez, de forma discriminada y acompañada de la significancia; como se ve, a menor longitud, mayor es el riesgo.

\section{Análisis multivariado de los factores de riesgo}

La única variable que en presencia de todas las demás presentó un valor estadísticamente significativo fue la longitud cervical por cervicometría menor de 30 mm con un OR 11,1 (IC95\% 4,55 - 27,05).

\begin{tabular}{|c|c|c|c|c|c|}
\hline \multicolumn{6}{|c|}{$\begin{array}{c}\text { en tres instituciones en } \\
\text { Medellín, Colombia - } 2004 \text {. }\end{array}$} \\
\hline Factor de riesgo & & Parto prematuro & Parto a término & Valor de $p$ & RR (IC95\%) \\
\hline Antecedente parto prematuro & Sí & 04 & 01 & $0,11 *$ & $5,05(0,54-46,4)$ \\
\hline $\mathrm{n}=134$ & No & 57 & 72 & & \\
\hline Tabaquismo & Sí & 03 & 00 & $0,09 * *$ & $2,20(1,81-2,67)$ \\
\hline $\mathrm{n}=124$ & No & 55 & 66 & & \\
\hline Infección urinaria & Sí & 09 & 16 & $0,22 *$ & $0,57(0,23-1,41)$ \\
\hline $\mathrm{n}=130$ & No & 52 & 53 & & \\
\hline Infección vaginal & Sí & 16 & 07 & $0,01 *$ & $3,22(1,22-8,48)$ \\
\hline $\mathrm{n}=129$ & No & 44 & 62 & & \\
\hline Dilatación > $10 \mathrm{~mm}$ & Sí & 12 & 07 & $0,09 *$ & $0,43(0,15-1,18)$ \\
\hline $\mathrm{n}=134$ & No & 49 & 66 & & \\
\hline Longitud $<30 \mathrm{~mm}$ & Sí & 41 & 12 & $0,00 *$ & $10,4(4,60-23,6)$ \\
\hline $\mathrm{n}=134$ & No & 20 & 61 & & \\
\hline
\end{tabular}

(*) Ji cuadrado

(**) Test de Fisher 


\section{DISCUSIÓN}

Una de las medidas más eficaces que ha logrado incidir sobre el pronóstico de los recién nacidos prematuros ha sido la aplicación de esteroides prenatales, los cuales, sin embargo, no están exentos de riesgos. Los fetos que más pueden beneficiarse de su administración son los hijos de gestantes con TPP idiopático.

No todas las embarazadas con síntomas presuntivos tienen un verdadero trabajo de parto ni el mismo riesgo de un parto prematuro; es así que uno de los principales dilemas diagnósticos en la práctica obstétrica moderna es el del TPP inicial. En el TPP inicial documentar los cambios cervicales por tacto vaginal es impreciso. Para evitar esta dificultad, varios autores han señalado la utilidad de la cervicometría en pacientes sintomáticas; Gómez y colaboradores ${ }^{11}$ evaluaron pacientes sintomáticas entre las 20 y 35 semanas de gestación con una dilatación cervical menor de $3 \mathrm{~cm}$ y encontraron una relación del parto prematuro con los hallazgos ecográficos, y no con los hallazgos al examen digital. Los autores sugieren la superioridad del primer método. También Iams ${ }^{12}$ evaluó 60 pacientes con síntomas de trabajo de parto pretérmino entre las 24 y 34 semanas, y en todos los casos las gestaciones que lograron el término tenían una cervicometría mayor a $30 \mathrm{~mm}$. Los resultados de esta investigación coinciden con estos hallazgos ya que se observó que una medida menor de $30 \mathrm{~mm}$ en la longitud cervical se constituyó en un factor de riesgo importante para el parto prematuro en este tipo de pacientes; de hecho, hubo una relación directa entre el acortamiento cervical y el incremento del riesgo (tabla 2).

La demostración de dilatación del orificio cervical interno por cervicometría no alcanzó significancia estadística y no demostró ser un factor de riesgo para padecer un parto prematuro en este estudio. Con relación a esto, otros autores encontraron que $70 \%$ de las pacientes sintomáticas entre las 20 y 35 semanas que tuvieron un parto prematuro tenían dilatación del OCI en la ecografía cervical transvaginal, a diferencia de las gestantes con parto a término, donde este hallazgo estuvo presente sólo en el 26\% ( $p<0,001)$; el promedio de longitud cervical fue $16,9 \pm 1,6 \mathrm{~mm}$ en las pacientes con parto prematuro. ${ }^{13}$

\begin{tabular}{|c|c|c|c|c|}
\hline \multirow{2}{*}{$\begin{array}{c}\text { Cervicometría } \\
\text { en mm }\end{array}$} & \multicolumn{2}{|c|}{ Parto prematuro } & \multirow{2}{*}{ Total } & \multirow{2}{*}{ OR $(*)$} \\
\hline & Sí & No & & \\
\hline$<15$ & 5 & 0 & 5 & 13,00 \\
\hline $15-25$ & 17 & 4 & 21 & 9,21 \\
\hline $26-35$ & 22 & 18 & 40 & 2,65 \\
\hline $36-45$ & 11 & 38 & 49 & 0,63 \\
\hline$>45$ & 6 & 13 & 19 & 1,00 \\
\hline Total & 61 & 73 & 134 & \\
\hline
\end{tabular}

(*) Ji cuadrado tendencia; $\mathrm{p}=0,00$

La embudización no se incluyó en los resultados de esta investigación. Este hallazgo es producto de un proceso progresivo de dilatación y acortamiento del cuello que para tener significancia clínica debe estar asociado con una longitud cervical residual corta, la cual se constituye en una medida más confiable y se correlaciona más consistentemente con la duración de la gestación. ${ }^{14}$

Otros factores de riesgo conocidos que estuvieron presentes en estas pacientes, como el antecedente de prematurez, tabaquismo, infecciones genitales, etc. no tuvieron significancia estadística y el riesgo dependió sólo del resultado de la cervicometría.

El presente estudio es el primero que se realiza en nuestro medio sobre el uso de la cervicometría para determinar el riesgo de parto prematuro en pacientes con sospecha clínica de "amenaza de parto prematuro". Esto es consistente con la literatura conocida, que señala que es poco probable que se presente un parto prematuro en pacientes sintomáticas, con membranas íntegras y dilatación menor de $3 \mathrm{~cm}$ antes de las 35 semanas de gestación 
en los 14 días posteriores a la observación mediante ecografía transvaginal de un cérvix con longitud mayor de $30 \mathrm{~cm}$; si la fibronectina en moco cervical es negativa, la probabilidad de parto prematuro es aún menor. ${ }^{15}$

Estos reportes indican el gran valor predictivo negativo de una cervicometría $>30 \mathrm{~mm}$ en gestantes con TPP inicial o "APP" (como se conoce en el medio) y el parto prematuro.

\section{REFERENCIAS}

1. Creasy RK, Resnik R, Iams JD, editors. Maternal-fetal medicine: principles and practices. Preterm labor and delivery. 5 ed. Philadelphia: Saunders; 2004.

2. Goldenberg RL. The management of preterm labor. Obstet Gynecol 2002;100:1020-37.

3. Tucker JM, Goldenberg RL, Davis RO, Cooper RL, Winkler CL, Hauth HC. Etiologies of preterm birth in an indigent population: is prevention a logical expectation? Obstet Gynecol 1991;77:343-7.

4. Meis PJ, Michielutte R, Peters TJ, Wells HB, Sands RE, Coles EC, et al. Factors associated with preterm birth in Cardiff, Wales. II. Indicated and spontaneous preterm birth. Am J Obstet Gynecol, 1995;173:597-9.

5. Meis PJ, Goldenberg RL, Mercer BM, Iams JD, Moawad AH, Miodovnik M, et al. The preterm prediction study: risk factors for indicated preterm birth. Maternal-Fetal Medicine Units Network of the National Institute of Child Health and Human Development. Am J Obstet Gynecol 1998;178:562-5.

6. Goldenberg RL, Iams JD, Mercer BM, Meis PJ, Moawad $\mathrm{AH}$, Copper RL, et al. The preterm prediction study: the value of new vs standard risk factors in predicting early and all spontaneous preterm births. NICHD MFMU Network. Am J Public Health 1998;88:233-8.

7. Newman RB. The Preterm Prediction Study: comparison of the cervical score and Bishop score for the prediction of spontaneous preterm birth. J Soc Gynecol Investig 1997;4:152-A.

8. Iams JD, Goldenberg RL, Meis PJ, Mercer BM, Moawad A, Des A, et al. The length of the cervix and the risk of spontaneous preterm delivery. National Institute of Child Health and Human Development Maternal Fetal Medicine Unit Network. N Engl J Med 1996;334:567-72.

9. The American College of Obstetricians and Gynecologists. ACOG practice bulletin. Assessment of risk factors for preterm birth. Clinical management guidelines for obstetrician-gynecologists. Number 31, October 2001. Obstet Gynecol 2001;98:709-16.

10. Cnattingius S, Granath F, Petersson G, Harlow BL. The influence of gestational age and smoking habits on the risk of subsequent preterm deliveries. N Engl J Med 1999;341:943-8.

11. Hillier SL, Nugent RP, Eschenbach DA, Krohn MA, Gibbs RS, Martin DH, et al. Association between bacterial vaginosis and preterm delivery of low birth-weight infant. The Vaginal Infections and Prematurity Study Group. N Engl J Med 1995;333:1737-42.

12. Gomez R, Galasso M, Romero R, Mazor M, Sorokin Y, Goncalves L, et al. Ultrasonographic examination of the uterine cervix is better than cervical digital examination as a predictor of the likelihood of premature delivery in patients with preterm labor and intact membranes. Am J Obstet Gynecol 1994;171:956-64.

13. Iams JD, Paraskos J, Landon MB, Teteris JN, Johnson FF. Cervical sonography in preterm labor. Obstet Gynecol 1994;84:40-6.

14. Timor-Tritsch IE, Boozarjomehri F, Masakowski Y, Monteagudo A, Chao CR. Can a "snapshot" sagital view of the cervix by transvaginal ultrasonography predict active preterm labor? Am J Obstet Gynecol 1996;174:990-5

15. Iams JD. Prediction and early detection of preterm labor. Obstet Gynecol 2003;101:402-12. 